\title{
Loi fédérale sur le dossier électronique du patient: nos efforts ont porté leurs fruits
}

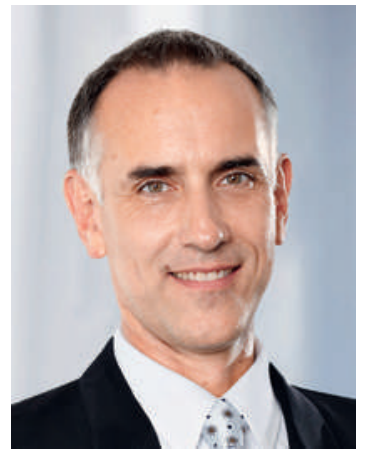

$\mathrm{Au}$ terme d'un processus de plusieurs années, le message relatif à la loi fédérale sur le dossier électronique du patient (LDEP) a été adopté le 29 mai 2013 par le Conseil fédéral à l'intention du Parlement.

Approuvée en 2007 par le Conseil fédéral et résultat de la collaboration entre la Confédération et les cantons, la stratégie Cybersanté Suisse se décline en trois volets: dossier médical informatisé, services en ligne et mise en œuvre. L'objectif poursuivi vise à étendre la «Stratégie pour une société de l'information en Suisse» de 1998 au domaine de la santé.

Pour la mise en œuvre de cette stratégie nationale, il s'est rapidement avéré essentiel de définir les conditions-cadres, des règles et des normes non seulement uniformes pour tout le pays et applicables au-delà des limites cantonales et des institutions de santé, mais aussi indépendantes des assureursmaladie. Dans ce contexte, les bases légales faisaient jusqu'à présent défaut. De plus, le cadre constitutionnel est plutôt fragile.

Désormais, la LDEP doit définir les exigences requises pour sécuriser le traitement des données dans le dossier électronique du patient, ainsi que les paramètres permettant de standardiser l'échange de données au niveau national. Concrètement,

- les patients auraient accès à leurs données médicales et pourraient décider qui est autorisé à les consulter et qui ne l'est pas. Toutes les données importantes seront disponibles en cas d'urgence;

- les médecins habilités par leurs patients auraient une vue d'ensemble des traitements déjà effectués, des résultats d'analyse et des médications du patient;

- via le rapport d'hospitalisation électronique, les hôpitaux auraient la possibilité d'impliquer en temps réel les médecins traitants, mais aussi le personnel soignant, de leur transmettre les informations importantes et d'avoir un retour sur l'impact de leurs interventions.

La FMH, qui a étroitement accompagné le processus d'élaboration du projet de loi, soutient la définition de conditionscadres uniformes pour un dossier électronique du patient mis en place à l'échelle nationale. Le groupe de travail eHealth de la FMH a passé en revue les différents aspects de la loi et a intensivement discuté des modalités possibles, pour certains points directement avec les représentants de l'OFSP. La FMH a systématiquement donné son avis lors de consultations, d'auditions et dans les groupes de travail. Un engagement qui a porté ses fruits puisqu'un grand nombre de nos demandes ont été reprises dans le présent projet de loi. Nous pouvons citer, par exemple, la règle relative au consentement dont la formulation est désormais acceptable, l'accès au dossier dont sont exclus les assureurs et le choix, tant pour le médecin que pour le patient, d'utiliser ou non un dossier électronique. Par ailleurs, en identifiant le patient indépendamment de son numéro AVS, on apporte une sécurité supplémentaire et on renforce la protection des données. Enfin, avec les aides financières prévues et la volonté de répercuter sur le tarif le traitement électronique des données on pourrait répondre aux souhaits de la FMH de créer les incitatifs judicieux.

\section{Plusieurs points essentiels défendus}

par le corps médical ont été repris dans le projet de loi.

Sous réserve du consentement préalable du patient, l'échange de données entre les médecins intervenants doit être garanti au-delà des limites cantonales et des structures de soins dans le respect de la confidentialité la plus absolue. La sécurité des données et l'autodétermination du patient sont aussi des facteurs déterminants pour l'acceptation d'un tel projet. Le dossier du patient doit être intégré dans le processus thérapeutique, mais il est également primordial d'associer les différents soignants à sa mise en œuvre.

L'efficacité de cette loi à créer un outil réellement utile à la prise en charge du patient dépendra de sa forme et réalisation finale mais aussi de ses modalités d'application sur lesquelles la FMH s'engagera à nouveau.

Dr Gert Printzen, membre du Comité central de la FMH, responsable du domaine Informatique médicale et cybersanté 